\title{
Beyond the Streetlight Effect: A United Future for Relaxation and Diffraction Methods for Residual Stress Measurement
}

\author{
Michael B Prime ${ }^{1}$, Michael L. Steinzig ${ }^{2}$ \\ PO Box 1663, Los Alamos National Laboratory, Los Alamos, NM 87544 USA \\ 1prime@lanl.gov, 2'steinzig@lanl.gov
}

\begin{abstract}
Keywords: Streetlight effect, superposition, combined methods, relaxation methods, contour method, hole drilling, diffraction.
\end{abstract}

\begin{abstract}
Residual stress measurement techniques can be categorized as either relaxation or diffraction methods. Practitioners often advocate a particular category and sometimes a specific technique (hole drilling, contour, XRD, neutron, etc) based on their experience or capability rather than using the best technique for the particular application. This paper considers some of the implications from applying this "drunkard's search" or "streetlight" approach by examining examples where the critical stress could be hidden from both relaxation and diffraction measurements. A better approach to planning residual stress measurements would begin with a detailed consideration of why the stresses should be measured and how the results will be used. Only then can the most appropriate measurement plan be developed. Since a single measurement technique cannot reveal the full state of stress, especially in challenging parts, the use of multiple measurement types often provides the most useful information to customers.
\end{abstract}

\section{Introduction}

This paper accompanies a Keynote lecture intended to introduce destructive measurement methods for residual stress. A recent review provides detailed information on new developments and future opportunities for destructive methods [1]. This paper instead takes a more holistic view of residual stress measurement, and therefore includes discussion of both destructive and nondestructive methods.

Prior to detailing the focus of this paper, we will describe some terminology. The majority of residual stress (RS) measurements can be categorized in two classes: relaxation techniques and diffraction techniques. These generally accepted terms describe the parameter that is measured. In the case of relaxation techniques, the stresses are somehow relieved, and the resulting relaxation of the material is measured. (Relaxation techniques are sometimes also referred to as mechanical methods, because of the mechanical action taken to remove material. In this discussion, we will not use the mechanical method terminology.) In the other case, the lattice spacing of the atomic structure is measured using a diffraction technique, and the measurement is based on the difference between the unstressed and stressed lattice spacing.

Relaxation techniques are often described as destructive, and diffraction techniques as nondestructive, but these descriptions are often misunderstood as applying to the condition of the part after the measurement is made, while they should more accurately be applied as corollaries to the descriptions of the technique: relaxation, which requires the material supporting the stress to be destructed so relaxation can occur, and diffraction, which does not require destruction of the supporting material. The misunderstanding occurs when applying the terms destructive and nondestructive to the work that is necessary to measure the stress, and the end condition of the part. While the application of a relaxation method necessarily destructs some material, (not necessarily the entire part), the application of a diffraction technique does not necessarily destruct material, but to obtain useful results from one of these techniques often does require destruction of parts. (Measurement of $\mathrm{d} 0$ for neutron diffraction, or material etching to allow X-ray diffraction access to 
deeper stresses are two examples of the destructive nature of supposedly non-destructive diffraction methods.)

With that introduction, our focus in this paper is to categorize RS measurements based on the motivation for the measurements, to describe some of the measurement opportunities and challenges in each of these categories, and to show some examples in which measurements from both classes of technique (relaxation and diffraction) are necessary for a complete picture of the RS that is needed for the analysis in question.

It is common that practitioners of residual stress measurement are often well versed in a measurement technique from one category (relaxation or diffraction), but have limited experience or aptitude for making measurements using other techniques. In some of the examples discussed, it will be clear that either the critical residual stress was not measured, or that the most appropriate technique for measuring the critical stress was not evaluated, which we attribute to some extent to the "streetlight" effect also known as the Drunkard's Search [2], where people do their searching where the looking is easiest, or in the case of RS measurement, where they have the most experience or comfort. The name for this effect comes from a joke:

A policeman finds a drunken man searching for something under a streetlight and asks what the drunk has lost. He says he lost his keys and they both look under the streetlight together. After a few minutes the policeman asks if he is sure he lost his keys here, and the drunk replies, no, that he lost them across the street. The policeman asks why he is searching here, and the drunk replies, "because the light is better here."

In defense of some of these measurements, it is common that the selection of measurement method is determined by a program leader (not by the measurement practitioner), but we assert that the streetlight effect commonly influences the decision by program personnel, and practitioners can play a role in helping to correct this phenomena.

\section{Looking Under the Streetlight}

This section looks at a few examples of "looking under the streetlight." In order to make the point, some examples from the literature are discussed overly simplistically. In general, researchers are aware of the limitations of their measurements and discuss all relevant issues. The measurements cited here are quite good work.

Currently, many larger parts are sliced, sectioned or otherwise altered in order to provide access and thereby exploit the capabilities of advanced neutron and synchrotron diffraction instruments $[3,4,5,6]$ or laboratory x-rays [7] or incremental hole drilling [8]. One common example is railroad rails that are cut into slices before RS measurement because they are too thick for neutrons or synchrotrons $[9,10,11,12,13,14,15]$. In general, the researchers are aware that the sectioning alters the stresses and treat the results appropriately, but many do not do anything to determine the important pre-sectioning stresses. Other techniques that can measure the residual stresses that existed prior to sectioning could be much more useful in such parts. Also, needless to say, the sectioning removes the "nondestructive" advantage of diffraction measurements.

Another common example that could be classified under the streetlight effect is measuring surface stresses when a bulk measurement would be more appropriate. Since the two most common residual stress measurement methods worldwide are probably incremental hole drilling and x-ray diffraction, one of those is often the most convenient tool to choose. Those methods are best used, for example, to measure stresses from some surface process such as machining, grinding, or shot peening. However, they are also often used to measures surface stresses on parts with important stresses through the thickness, such as welds. Often such measurements are used to validate or calibrate a finite element process model. Bulk measurements would often be more appropriate to calibrate the model using peak stress regions and including subsurface regions where stresses are triaxial. Furthermore, using a surface stress measurement can be misleading. Often the surface has stresses not only from the process that is being modeled, but from other processes that are not 
modeled, such as machining operations used in forming the blank part. Calibrating a process model on stresses from a different process would be woefully inadequate.

\section{Looking Where it Matters - Selecting an Appropriate Measurement Method}

In general, no measurement method can measure all components of stress throughout a threedimensional part. A selection must be made. Guidance often comes from comparing different aspects of measurement methods, such as depth of penetration, and a recent handbook provides up to date information [16] on such parameters.

However, the first and most critical but often least discussed step in selecting a measurement method should be to answer the overall question why are the stresses being measured in the first place? Only then can the appropriate method(s) be selected. Once the overall reason for the measurement has been carefully considered and elucidated, some underlying questions that should be answered to select a method are:

1. Which component(s) of stress needs to be measured?

2. Where in the part do you want to measure residual stress?

3. Do you need a point measurement, a stress profile, or a 2-D or 3-D map?

4. How much spatial resolution is needed?

5. Which criteria can be relaxed? With current limitations on measurement techniques, meeting all the criteria for the measurement is not likely.

As the first example of several measurement categories, we consider the category of measurements that deal with structural integrity, where the answer to the why question is to allow prediction of the initiation and growth of cracks from fatigue or stress corrosion cracking. The underlying questions are answered in the following discussion:

1. The stress component that needs to be measured in this case is normal to the direction of the crack, since that is the component that drives the crack growth. This is a straightforward answer, although in practice it is often a hypothetical crack being considered, in which case the stress that needs to be measured is in the worst case direction, which is generally the direction of the maximum tensile stress.

2. The stress should be measured in the most likely failure location(s), which is a combination of high tensile stress locations and location where cracks will likely initiate, including surfaces exposed to hostile environments, geometrically influenced stress risers, and interfaces where metallurgy changes may provide additional susceptibility to corrosion.

3. To answer questions 3 and 4, questions 1 and 2 would need definitive answers. Crack growth predictions generally require estimating the stress intensity factor, $K_{I}$, from residual stresses, which requires knowledge of the residual stresses over the plane that will contain the crack. A point measurement is rarely sufficient. If the stresses do not vary significantly over the transverse dimensions a 1-D depth profile down to the maximum crack depth of interest can be sufficient. A 2-D map over the crack plane is required when the stresses vary in the transverse dimensions. 3-D information might be desired when the location of a hypothetical crack is unknown, but is rarely practical to obtain.

4. Resolution needs to be sufficient to quantify the spatially-integrated effect of all the released residual stresses on $K_{I}$. The resolution should ideally be some fraction of the maximum crack depth or small enough to approximately resolve relevant gradients.

5. The first criteria to relax would be any requirement for 3-D stress mapping, since it is so challenging. Alternate approaches will be discussed later.

The choice of methods for such structural integrity issues is thus dependent on the crack location being considered. Often x-ray diffraction, hole drilling and incremental slitting are good choices for surface crack locations. Subsurface crack locations might require neutron diffraction, deep hole drilling, or contour measurements. 
Another very common category of measurements is those used to validate some process model, such as a finite element model. A cursory examination of this category might lead a practitioner to the conclusion that no restrictions on the measurement technique is necessary, since in principle a specific stress location or component is not needed to validate a model. Good choices might be where the largest stresses are. A better choice might be measuring the stresses that are most sensitive to modeling unknowns. A more detailed examination of the problem leads to the understanding that the final state of residual stress is the endpoint of some complicated thermomechanical process, for example casting followed by forging and a final welding process. If the model does agree with the measured results, it is not a guarantee that the model is correct rather than a fortuitous coincidence. If the model does not agree with the measurements, it is very difficult to know where during the process the model went wrong. Adjusting the model to match the endpoint is potentially a non-unique inverse problem, and it is likely that you end up without a very predictive model. This points out a potentially underutilized capability for validating process models. An in situ measurement using neutrons or synchrotrons can measure stresses during the process rather than just at the endpoint $[17,18,19]$. Although such measurements are currently only feasible under limited conditions, it is the most powerful way to validate a model. A good approach might be to perform in situ measurements on smaller test coupons that undergo different portions of the processing before a validation of the final residual stresses in a full component.

A third category is measurements used for certification of a part or structure for some important application, maybe even a life critical application. In this case, high confidence in the results is required. In such cases, it is most wise to employ two independent measurements. Here, independence means that the different measurement methods must use different physical principles with different underlying assumptions to measure RS. In that way, it is nearly impossible to get the same incorrect, biased answer from both techniques. The most common and most effective way to achieve the independent measurements is to use one relaxation method and one diffraction method.

A fourth category is measurement for quality control purposes. The why in this case is to verify that a process has not changed or broken down resulting in a detriment to part quality. Prior to evaluating the different types of techniques available for the quality control measurement, a significant effort to determine the full state of stress and the expected stress variation in the part should probably have been completed. This will allow for the most efficient and appropriate measurement technique to be selected to provide quality control. Typically this type of measurement must be rapid, automated, useable in a production environment, nondestructive and in many cases does not have to be fully quantitative. Techniques like magnetic methods or ultrasonics, which are neither relaxation not diffraction methods, may be ideal.

Other categories that have different measurement considerations include part distortion issues, creep cracking, and forensic failure analysis [20]. Because of space limitations, those are not discussed here.

An important consideration for some purposes is the need for a 3D map of the full residual stress tensor for use in finite element models for some predictions. If an initial residual stress state is used in the model, the full tensor must be defined everywhere, or else the finite element code will make various assumptions in order to achieve an admissible initial state. Measurement of full 3-D stress maps is impractical. So in such cases, eigenstrain analyses [21, 22] and other similar numericalexperimental approaches $[23,24]$ use the available measured data plus physical constraints (such as equilibrium and compatibility) to determine the most likely stress map. Even when a full 3D stress map is not needed (which is often the case if one uses an intelligent approach), such methods are still necessary to expand limited measurement data to cover the region where stresses are needed.

\section{Examples of Measurement Challenges}

The doctrine that fatigue cracks always start on a surface has sometimes led to an over-emphasis on near-surface measurements of residual stress, such as using x-ray or hole drilling. Although the 
majority of fatigue cracks probably start on a surface, many do not. An illustrative example is the railroad industry in the USA [25]. Figure 1 a) and b) show fatigue cracks that initiated subsurface. According to an expert at the USA Department of Transportation, "Subsurface-initiated cracks are the bane of the industry and are the cause of $35 \%$ of freight train derailments [25]." A previous section of this paper described the multitude of measurements done with diffraction on slices removed from rails, where the original stresses have partially relaxed. By contrast, Figure 1c shows a contour method measurement [26] that showed the subsurface residual tensile stress that leads to such cracks [27], measured in the full component.
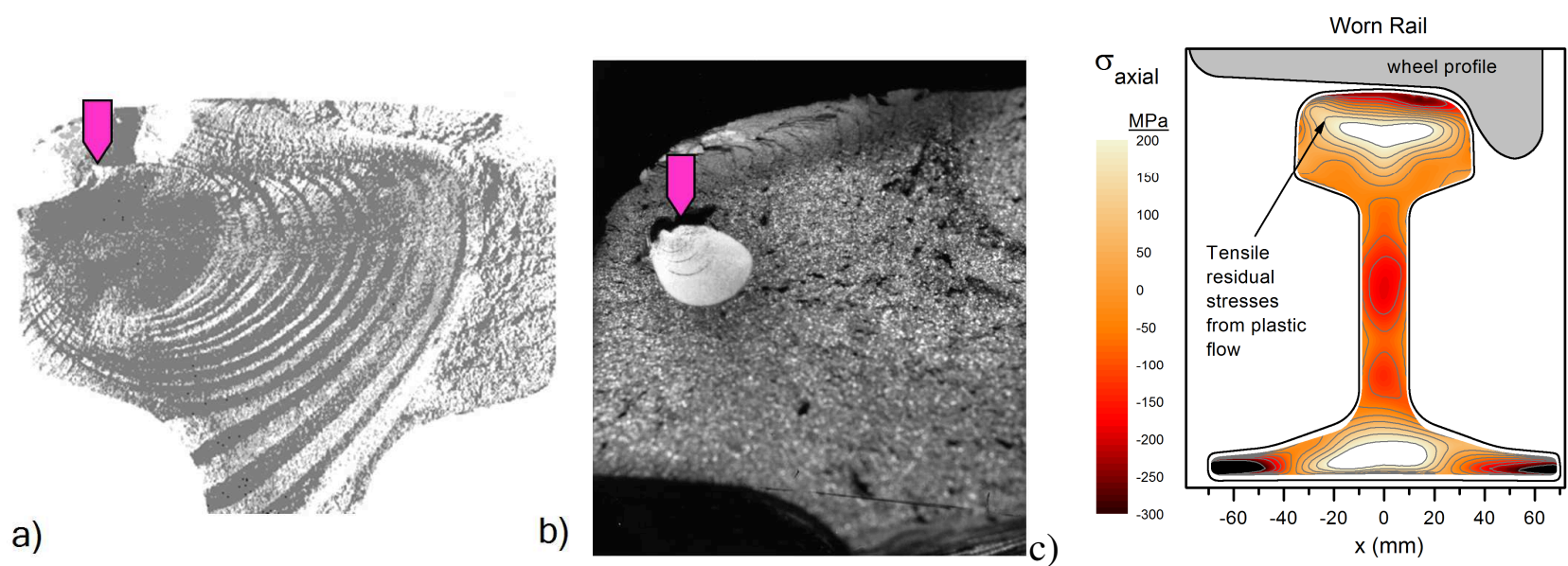

Figure 1. Fatigue cracks that initiated subsurface on a) normal USA rail and b) a rail ground for 2-point contact. c) A contour method measurement on UK rail showing the subsurface longitudinal tensile residual stress.

There is reason to be concerned that the incidence of subsurface fatigue cracking will increase. Many manufacturers are taking advantage of relatively new compressive residual stresses generated by Laser Shock Peening [28] and Low Plasticity Burnishing [29]. These methods offer increased fatigue crack resistance because they impart a deeper compressive stress layer than shot peening and other older technologies. However, deeper compressive stresses means there must be balancing tensile stresses somewhere, and more than there would be for shallower compressive stress treatments, which increases the concern for fatigue cracks initiating subsurface or in some other unexpected region $[30,31]$.

Another example to be considered is the distinction between the average, or steady state condition, and the associated residual stress, and the worst case residual stress associated with a transient processing condition. For example, in the case of a weld, it would be common to characterize the residual stress at a steady state condition of the weld, even though the transient conditions at either a weld schedule power change, or the weld stop/start may result in a localized maximum for tensile residual stress [32]. If this condition of maximum residual stress also happens to be co-located at a critical position, such as at a geometrical stress concentration, or on the inner diameter of a pressure vessel, the residual stress condition may now be doubly difficult for the RS practitioner to quantify. In the case of a circumferential weld around a pressure vessel or pipe, the entire weld may have to be scanned to locate the condition of maximum residual stress, and the measurements must be made on the inside of the vessel, which presents the most difficult access problem. In some cases, this measurement can be simplified if the location of the weld transients can be identified and targeted for measurement.

One major category of difficult problems is mapping internal stresses in welded components that are too thick for neutron diffraction. A significant example is circumferential welds in large components for nuclear power plants, where welding residual stresses are a major concern [33]. Some approaches to solving this category of problems are discussed next. 


\section{New Novel Approaches to Challenges}

Some very recent work has exploited the capabilities of the contour method [26] and added unique diffraction capabilities in order to map stresses in thick parts. First a conventional contour method measurement is made: the part is sectioned to map the RS component normal to the cut. The limitation with the contour method is that only the one stress component is determined. However, the contour measurement also exposes a new surface that is now accessible to surface measurement (for example by x-ray diffraction or hole drilling). The surface measurement can be combined with the contour result to determine the original state of residual stress. This multiple-methods superposition theory was originally proved using a specimen that was small enough to independently measure with neutron diffraction [34]. More recently, the method has been used to map multiple stress components inside a challenging, large, circumferentially welded pipe, as shown in Figure 2 [35].
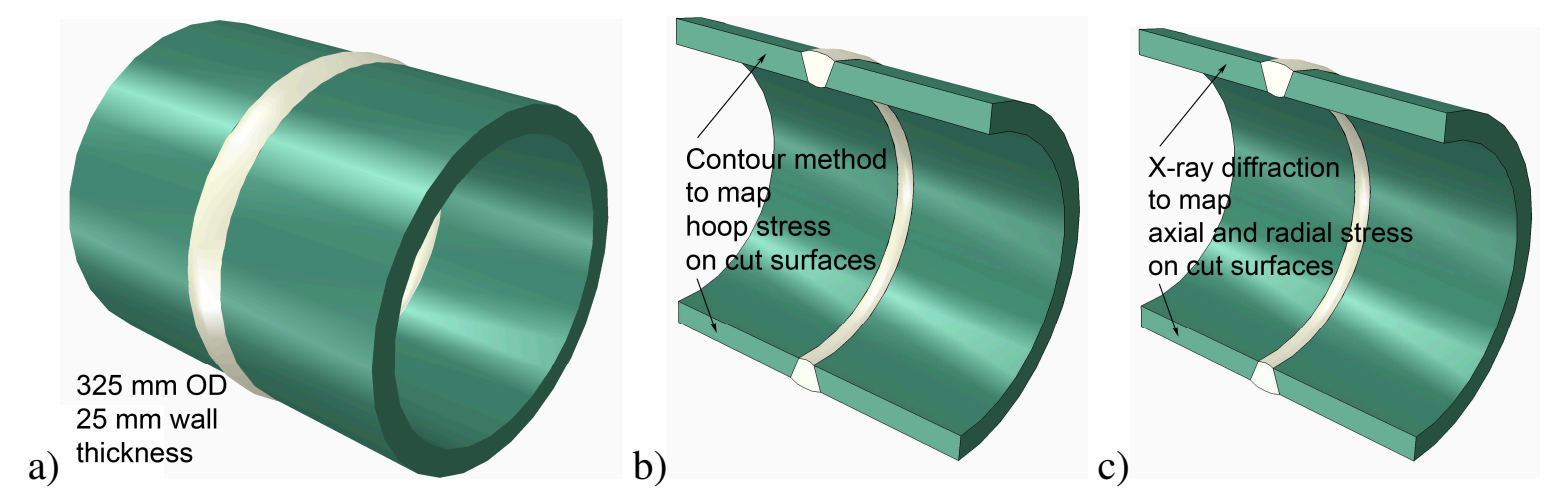

Figure 2. a) a large circumferentially-welded steel cylinder was b) cut in half to measure the hoop stresses then c) the radial and axial stresses were measured with $\mathrm{X}$-ray diffraction and then combined with contour results to reconstruct the original stresses prior to cutting.

The approach in the previous paragraph, contour method plus diffraction, has so far only been used with a single contour cut and then surface stress measurements. In principle, the same approach could be used with two cuts to cut out a slice. One could then use advanced mapping capabilities with synchrotron or neutron diffraction to reconstruct a full map of the full stress tensor. This approach would be appropriate for the slice measurements, such as railroad rails, discussed earlier in this paper.

A recent publication details a comprehensive set of measurements in order to map residual stresses in a very difficult part: a large, thick-walled nozzle from a nuclear power plant and made with dissimilar metals [36]. Those measurements used an impressive sequence of multiple relaxation and diffraction techniques: splitting, contour method, sectioning with Digital Image Correlation (DIC), and then neutron diffraction in the slice. Other recent work has used multiple destructive measurements, including slitting [37], and superposition to extend the capability of destructive methods [38].

Some other recent work combining two destructive methods has resulted in other advanced capabilities. In contrast to the contour method, the deep hole method [39] measures only a 1-D profile of RS, but it does measure all three in-plane stress components. Deep hole has been applied to nuclear components up to $735 \mathrm{~mm}$ inner diameter and $73 \mathrm{~mm}$ wall thickness [40], bigger than any other method. Like the contour method, deep hole drilling has difficulty resolving RS near the surface. In a rather ingenious insight, incremental hole drilling was first used in a large component to measure the near-surface stresses. A subsequent deep hole measurement was made using a larger diameter hold and completely removing the initial hole. Since the reference data for deep hole is taken after the through-hole is drilled, the smaller incremental hole has no effect and the two measurements combine to reveal the full stress profile [41]. 
Other deep hole work on similar thick weldments has combined deep hole with X-ray diffraction and numerical analyses to obtain the required stresses [42].

\section{Conclusions}

Doing the best job of determining the residual stresses that are important for some purpose requires several considerations. A thorough understanding of why one wants to know the residual stresses and how they will be used is paramount. Parochial preferences must then be abandoned in order to determine the best way to measure the stresses, and the most sensible approach will often make use of multiple measurement techniques from both the destructive and non-destructive methods.

\section{References}

[1] G.S. Schajer, Relaxation Methods for Measuring Residual Stresses: Techniques and Opportunities, Experimental Mechanics, 50 (2010) 1117-1127.

[2] Abraham Kaplan, The Conduct of Inquiry: Methodology for Behavioral Science, Harper \& Row, 1964

[3] S.V. Pearce, V.M. Linton, E.C. Oliver, Residual stress in a thick section high strength T-butt weld, Materials Science \& Engineering: A, 480 (2008) 411-418.

[4] A. Stacey, H.J. MacGillivary, G.A. Webster, P.J. Webster, K.R.A. Ziebeck, Measurement of residual stresses by neutron diffraction, Journal of Strain Analysis for Engineering Design, 20 (1985) 93-100.

[5] U. de Oliveira, V. Ocelik, J.T.M. De Hosson, Residual stress analysis in Co-based laser clad layers by laboratory $\mathrm{X}$-rays and synchrotron diffraction techniques, Surface and Coatings Technology, 201 (2006) 533-542.

[6] L. Djapic Oosterkamp, P.J. Webster, P.A. Browne, G.B.M. Vaughan, P.J. Withers, Residual stress field in a friction stir welded aluminium extrusion, Materials Science Forum, 347-349 (2000) 678-683.

[7] E.J. McDonald, K.R. Hallam, W. Bell, P.E.J. Flewitt, Residual stresses in a multi-pass CrMoV low alloy ferritic steel repair weld, Materials Science and Engineering A, 325 (2002) 454-464.

[8] H. Jahed, M.R. Faritus, Z. Jahed, Residual Stress Measurements in an Autofrettage Tube Using Hole Drilling Method, Journal of Pressure Vessel Technology, 134 (2012) 051501-051501.

[9] P.C. Brand, H.J. Prask, T. Gnaeupel-Herold, Residual stress measurements at the NIST reactor, Physica B, 241-243 (1997) 1244-1245.

[10] T. Sasaki, S. Takahashi, Y. Kanematsu, Y. Satoh, K. Iwafuchi, M. Ishida, Y. Morii, Measurement of residual stresses in rails by neutron diffraction, Wear, 265 (2008) 1402-1407.

[11] D. Stefanescu, P. Browne, C.E. Truman, D.J. Smith, Residual stress measurement within a European UIC60 rail using integrated drilling techniques, Materials Science Forum, 440-441 (2003) 85-92.

[12] D. Tawfik, O. Kirstein, P.J. Mutton, C. Wing Kong, Verification of residual stresses in flashbutt-weld rails using neutron diffraction, Physica B, 385-386 (2006) 894-896. 
[13] D.J. Buttle, N. Collett, P.J. Webster, D.J. Hughes, G. Mills, A comparison of MAPS and synchrotron X-ray methods: stresses measured in railway rail sections, Materials Science Forum, 404-407 (2002) 881-886.

[14] P.J. Webster, D.J. Hughes, G. Mills, G.B.M. Vaughan, Synchrotron X-ray measurements of residual stress in a worn railway rail, Materials Science Forum, 404-407 (2002) 767-772.

[15] T.S. Jun, F. Hofmann, J. Belnoue, X. Song, M. Hofmann, A.M. Korsunsky, Triaxial residual strains in a railway rail measured by neutron diffraction, The Journal of Strain Analysis for Engineering Design, 44 (2009) 563-568.

[16] G.S. Schajer and C.O. Ruud, C. O. (2013) Overview of Residual Stresses and Their Measurement, in: G. S. Schajer (Ed.) Practical Residual Stress Measurement Methods, John Wiley \& Sons, Ltd, Chichester, UK, 2013, pp. 1-7. doi: 10.1002/9781118402832.ch1

[17] W. Woo, et al., In situ neutron diffraction measurements of temperature and stresses during friction stir welding of 6061-T6 aluminium alloy, Science and Technology of Welding \& Joining 12 (2007) 298-303.

[18] A. Kromm, T. Kannengiesser and J. Gibmeier, In Situ Studies of Phase Transformation and Residual Stresses in LTT Alloys During Welding Using Synchrotron Radiation, in T. Kannengiesser et al. (Eds.), In-situ Studies with Photons, Neutrons and Electrons Scattering, Springer Berlin Heidelberg, 2010, pp. 13-26.

[19] B. Chen, A. Skouras, Y.Q. Wang, J.F. Kelleher, S.Y. Zhang, D.J. Smith, P.E.J. Flewitt, M.J. Pavier, In situ neutron diffraction measurement of residual stress relaxation in a welded steel pipe during heat treatment, Materials Science and Engineering: A, 590 (2014) 374-383.

[20] M.B. Prime, A.T. DeWald, M.R. Hill, B. Clausen, M. Tran, Forensic Determination of Residual Stresses and KI from Fracture Surface Mismatch, Engineering Fracture Mechanics, 116 (2014) 158-171.

[21] A.M. Korsunsky, Eigenstrain analysis of residual strains and stresses, The Journal of Strain Analysis for Engineering Design, 44 (2009) 29-43.

[22] A.T. DeWald, M.R. Hill, Eigenstrain based model for prediction of laser peening residual stresses in arbitrary 3D bodies. Part 1: model description, Journal of Strain Analysis for Engineering Design, 44 (2009) 1-11.

[23] S.A. Faghidian, D. Goudar, G.H. Farrahi, D.J. Smith, Measurement, analysis and reconstruction of residual stresses, The Journal of Strain Analysis for Engineering Design, 47 (2012) 254-264.

[24] Y.Z. Ge, M.A. Sutton, X. Deng, A.P. Reynolds, Limited weld residual stress measurements in fatigue crack propagation: Part I. Complete field representation through least-squares finite-element smoothing, Fatigue \& Fracture of Engineering Materials \& Structures, 29 (2006) 524-536.

[25] J. Gordon, Residual Stresses and Failures in Railroad Rail and Wheels: Experimental and Analytical Techniques, Presented at the 2005 Residual Stress Summit, Vancouver, BC, Canada, August 10-11, 2005.

[26] M.B. Prime, A.T. DeWald, The Contour Method, in: G.S. Schajer (Ed.) Practical Residual Stress Measurement Methods, John Wiley \& Sons, Ltd., Chichester,WestSussex, UK, 2013.

[27] J. Kelleher, M.B. Prime, D. Buttle, P.M. Mummery, P.J. Webster, J. Shackleton, P.J. Withers, The Measurement of Residual Stress in Railway Rails by Diffraction and Other Methods, Journal of Neutron Research, 11 (2003) 187-193. 
[28] A.H. Clauer, Laser shock peening for fatigue resistance, Surface Performance of Titanium, JK Gregory, et al, Editors, TMS Warrendale, PA, (1996) 217-230.

[29] P.S. Prevéy, J.T. Cammett, The influence of surface enhancement by low plasticity burnishing on the corrosion fatigue performance of AA7075-T6, International Journal of Fatigue, 26 (2004) 975-982.

[30] M. Dorman, M.B. Toparli, N. Smyth, A. Cini, M.E. Fitzpatrick, P.E. Irving, Effect of laser shock peening on residual stress and fatigue life of clad 2024 aluminium sheet containing scribe defects, Materials Science and Engineering: A, 548 (2012) 142-151.

[31] K.K. Liu, M.R. Hill, The effects of laser peening and shot peening on fretting fatigue in Ti6Al-4V coupons, Tribology International, 42 (2009) 1250-1262.

[32] A.M. Paradowska, J.W.H. Price, T.R. Finlayson, U. Lienert, R. Ibrahim, Comparison of Neutron and Synchrotron Diffraction Measurements of Residual Stress in Bead-on-Plate Weldments, Journal of Pressure Vessel Technology, 132 (2010) 011502-011502.

[33] S.H. Bush, Failure Mechanisms in Nuclear Power Plant Piping Systems, Journal of Pressure Vessel Technology, 114 (1992) 389-395.

[34] P. Pagliaro, M.B. Prime, J.S. Robinson, B. Clausen, H. Swenson, M. Steinzig, B. Zuccarello, Measuring Inaccessible Residual Stresses Using Multiple Methods and Superposition, Experimental Mechanics, 51 (2011) 1123-1134.

[35] F. Hosseinzadeh, P. Bouchard, Mapping Multiple Components of the Residual Stress Tensor in a Large P91 Steel Pipe Girth Weld Using a Single Contour Cut, Experimental Mechanics, 53 (2012) 171-181.

[36] M.R. Hill, M.D. Olson, Biaxial Residual Stress Mapping in a PWR Dissimilar Metal Weld, ASME 2013 Pressure Vessels and Piping Conference. American Society of Mechanical Engineers, 2013, paper PVP-2013-97246.

[37] M.R. Hill, The Slitting Method, in: G.S. Schajer (Ed.) Practical Residual Stress Measurement Methods, John Wiley \& Sons, Ltd, 2013, pp. 89-108.

[38] W. Wong, M.R. Hill, Superposition and Destructive Residual Stress Measurements, Experimental Mechanics, 53 (2013) 339-344.

[39] D.J. Smith, Deep Hole Drilling, in: G.S. Schajer (Ed.) Practical Residual Stress Measurement Methods, John Wiley \& Sons, Ltd, 2013, pp. 65-87.

[40] K. Ogawa at al. Measurement of residual stresses in the dissimilar metal weld joint of a safeend nozzle component, Proceedings of PVP2009 ASME Pressure Vessels and Piping Division Conference July26-30, 2009, Prague,Czech Republic, Paper PVP2009-77830.

[41] D. Stefanescu, C.E. Truman, D.J. Smith, An integrated approach for measuring near-surface and subsurface residual stress in engineering components, The Journal of Strain Analysis for Engineering Design, 39 (2004) 483-497.

[42] A.H. Yaghi, G. Hilson, S. Simandjuntak, P.E.J. Flewitt, M.J. Pavier, D.J. Smith, T.H. Hyde, A.A. Becker, W. Sun, A Comparison Between Measured and Modeled Residual Stresses in a Circumferentially Butt-Welded P91 Steel Pipe, Journal of Pressure Vessel Technology, 132 (2010) 011206-011206. 